\title{
Dose Load during Angiographic Procedures
}

\author{
Natasha Ivanova1, Javor Ivanov ${ }^{2}$ \\ ${ }^{1}$ Department of Physics and Biophysics, Medical University, Varna, Bulgaria \\ ${ }^{2} \mathrm{~S} \& \mathrm{~T}$ Bulgaria EOOD, Sofia, Bulgaria \\ Email: natasha_i@abv.bg
}

How to cite this paper: Ivanova, N. and Ivanov, J. (2021) Dose Load during Angiographic Procedures Open Access Library Journal, 8: e7109.

https://doi.org/10.4236/oalib.1107109

Received: December 22, 2020

Accepted: February 19, 2021

Published: February 22, 2021

Copyright $\odot 2021$ by author(s) and Open Access Library Inc.

This work is licensed under the Creative Commons Attribution International License (CC BY 4.0).

http://creativecommons.org/licenses/by/4.0/

\begin{abstract}
On basis of dosimetric measurement, this article compares the radiation doses received by the main interventional cardiologist during the angiographic procedures. The dose load during three procedures is compared-LAD-stenting, LCx-stenting and RCA-stenting. An angiographic X-ray system Philips Allura Xper FD10 (with G-arm) was used. The dose obtained was measured with an X-ray-Gamma Dosimeter 27091. During each procedure, two modes of the equipment are used-radiography and fluoroscopy. The dose measurements were made for the respective projections of each angiographic procedure and for the operation modes of the equipment used during the respective procedure at three measurement points on the operator's body-head, gonads and feet and at three position of the patient's table-lowest, highest and zero. The results obtained from the calculations, based on the measured dose values, show maximum dose load in the procedure that uses the radiographic mode of operation for the longest time, namely RCA-stenting. The other two procedures have the same time on radiography and fluoroscopy. From them a higher dose load was obtained for the procedure LAD-stenting. Of the three procedures studied, the lowest dose load is obtained for the procedure LCx-stenting.
\end{abstract}

\section{Subject Areas \\ Internal Medicine}

\section{Keywords}

G-Arm Type Angiographic X-Ray System, Dose Load, Interventional Cardiology, Cardiology Hospital

\section{Introduction}

In the modern world, the use of X-ray equipment for diagnostic and treatment purposes is increasingly affecting our lives. Because of this, safety is a constant 
concern when using this type of equipment. This was the motivation for our team to focus the study on the dose load of the staff working with X-ray equipment. The other major reason was the interventional cardiologists' interest in knowing the radiation doses they are receiving during the procedures.

We found the following interesting articles on this topic:

Keith J. Strauss and J. Anthony Seibert in "Angiographic Equipment Selection and Configuration" discuss how to choose the accessories and the right equipment, depending on the activities for which it is intended to be used. Equipment should be tailored to the needs of patients imaged in the angiography suite and the received dose. The basic management of these components, for example, optimization of radiographic techniques used during the acquisition of images from the production of X-rays, is briefly discussed [1].

Patient dose is discussed by V. Sadick, W Reed, et al. in "Impact of biplane versus single-plane imaging on radiation dose, contrast load and procedural time in coronary angioplasty". Coronary angioplasties can be performed with either single-plane or biplane imaging techniques. The aim of this study is to determine whether biplane imaging, in comparison to single-plane imaging, reduces radiation dose and contrast load and shortens procedural time during 1) primary and elective coronary angioplasty procedures, 2) angioplasty to the main vascular territories and 3) procedures performed by operators with various levels of experience. This prospective observational study included a total of 504 primary and elective single-vessel coronary angioplasty procedures utilising either biplane or single-plane imaging. Radiographic and clinical parameters were collected from clinical reports and examination protocols. Radiation dose is measured by a dose-area-product (DAP) meter intrinsic to the angiography system. The results show that biplane imaging delivered a significantly greater radiation dose $\left(181.4 \pm 121.0 \mathrm{Gycm}^{2}\right)$ than single-plane imaging (133.6 \pm 92.8 $\left.\mathrm{Gycm}^{2}, \mathrm{p}<0.0001\right)$. Biplane imaging resulted in a greater radiation dose and a longer procedural time and delivered a non-significant reduction in contrast load than single-plane imaging [2].

Mavrikou I, Kottou S, et al. in "High patient doses in interventional cardiology due to physicians' negligence: how can they be prevented?" discussed patient dose, too. Interventional cardiology procedures are usually associated with high patient doses and even deterministic radiation effects may occur. Expensive digital flat panels are preferably used to lower doses, and Athens General Hospital has recently installed one. However, this study shows that it is the cardiologists' practice that lowers patients' doses. Doses delivered to patients during two time periods (pre and after radiation protection training) on a total of 1196 coronary angiographies and 506 percutaneous transluminal coronary angioplasties were measured and analyzed per cardiologist. Local reference levels (LRLs) are assessed and compared with the preliminary RLs provided by the European Research Program DIMOND. Results show that although after the training patients' dose area product, fluoroscopy time, cumulative dose and number of images acquired were lowered, the situation remained unchanged for the cardiolo- 
gist who delivered the highest doses. The question to answer next is how this bad practice can be prevented since no dose constraints apply to diagnostic or therapeutic procedures using ionizing radiation [3].

In the article " 5 Technologies to Reduce Cath Lab Radiation Exposure" its authors discuss reducing of the staff radiation dose from X-ray angiography and long-term back pain due to weight of lead aprons. In the past few years, concern has skyrocketed from interventional cardiologists and cath lab staff over radiation dose exposure from the angiographic X-ray imaging systems. This is partly due to accumulation of study data showing the impact of radiation exposure on the job, with interventional cardiologists having higher rates of left-sided brain tumors, skin cancer, posterior subcapsular lens changes (a precursor to cataracts), thyroid disease and neuro-degenerative disease. Additionally, wearing heavy lead aprons over the course of their careers, interventional cardiologists suffer higher rates of orthopedic back pain issues [4].

All of these articles discuss the effective use of the devices in order to reduce as much as possible the dose received by the patient and by the staff working with the equipment.

Continuing the position to reduce the dose received, we performed measurements of the dose received by the first cardiologist-operator during invasive cardiac procedures.

\section{Study}

\subsection{Purpose of the Study}

The purpose of the study was based on the performed dosimetric measurements to make evaluation of the dose load, received by an interventional cardiologist from to the scattered X-ray radiation for different angiographic procedures during work with the angiographic system Philips Allura Xper FD10 [5].

\subsection{Motivation}

The specific angiographic procedures require use of different projections where the X-ray tube is pointed at a specific angle to the patient table and to the main cardiologist, performing the procedure. During each procedure several different projections are used. For each projection a specific dose load is received by the main interventional cardiologist. In some projections, both fluorography and radiography modes of operation of the X-ray equipment are used and the exposure time for the respective projection and operation mode is different as well. Thus, the total dose load for each specific angiographic procedure is different. All these circumstances inspired us to perform calculations based on the data collected from the measurements and to estimate the dose load that the main operator receives during each specific procedure [5].

\subsection{Methods}

\section{Dosimetric measurement}


We measured the dose received by a cardiologist performing an angiographic procedure. Measurements were performed for all of the 9 most commonly used G-arm projections. Each measurement was made at three points on the cardiologist's body: head, gonads and feet and for three different table positions: zero height (according to the default system adjustment), lowest possible position for the respective projection and highest possible position [5].

\subsection{Practical Measurements}

The practical measurements were made in the Specialized Hospital for Active Treatment in Cardiology (SBALK) Varna, in the Department of Invasive Cardiology [5].

\subsubsection{Participants}

Participants in the dosimetric measurements:

- the first author of this article-a representative of the Medical University and processing the received data,

- service engineer maintaining the X-ray equipment: the measurements were carried out under the direct and continuous control of the service engineer of the X-ray system,

- medical physicist-Head of the Radiation Protection Department at the Regional Health Inspectorate, Varna, working with the measuring device,

- three interventional cardiologists from the Cardiac Hospital who provided information and instructions on the interventional procedures and projections used during the measurements [5].

\subsubsection{Devices and Materials}

The study was performed in a procedure room of an angiographic X-ray system with G-arm Philips Allura Xper FD10 (Figure 1) [5].

For the dosimetric measurement was used a X-Ray-Gamma-Dosimeter RGD 27091 (Figure 2) [5] [6].

A 19-liter water bottle was used as phantom (Figure 3) [5].

\subsubsection{Practical Work Procedure}

The dose measurements were performed for three different patient table positions-zero height (default position set by the factory), highest and lowest position for the respective procedure for the three measurement points- "Head", "Gonads", "Feet". Figure 4 shows the positions of the three measurement points.

Dose evaluation of the first operator was performed for three angiographic procedures: LAD-stenting, LCX-stenting and RCA-stenting.

1) ALD-stenting. Following projections are used:

Total time for the performed procedure 45 - $90 \mathrm{~min}$ (Table 1).

Total time for the basic projection 30 to 60 minutes: Each of the three mentioned projections can be used as basic projection. The exposure time in pulse fluoroscopy mode for the basic projection is about $10 \mathrm{~min}$. The exposure time in radiography mode for the basic projection is about $2-3 \mathrm{~min}$. 


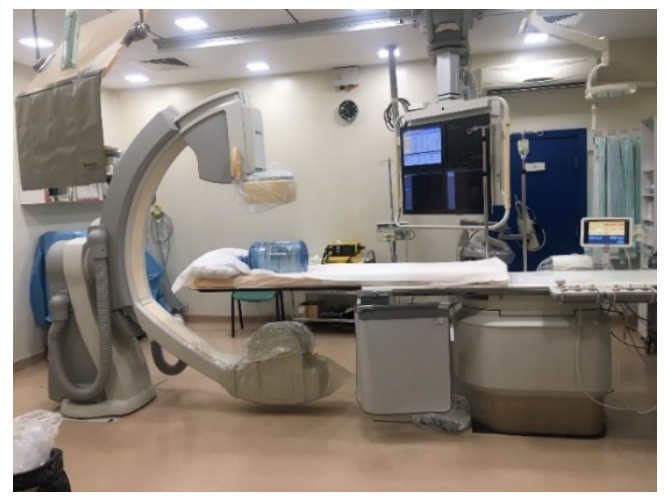

Figure 1. Angiographic X-ray system Philips Allura Xper FD10.

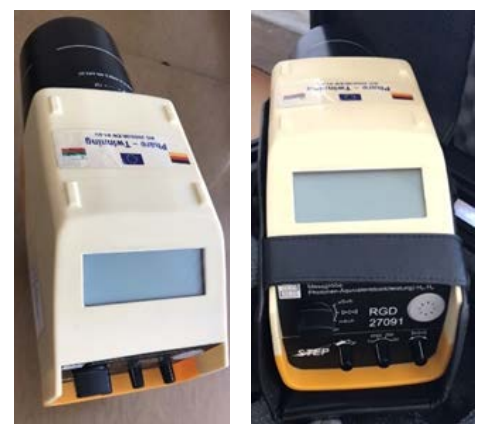

Figure 2. X-Ray-Gamma-Dosimeter RGD 27091.

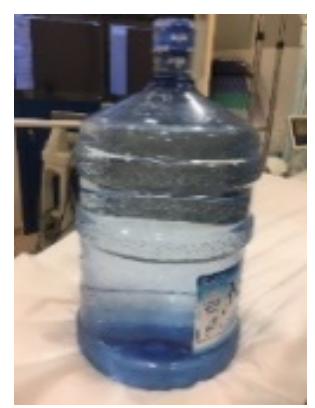

Figure 3. Phantom.

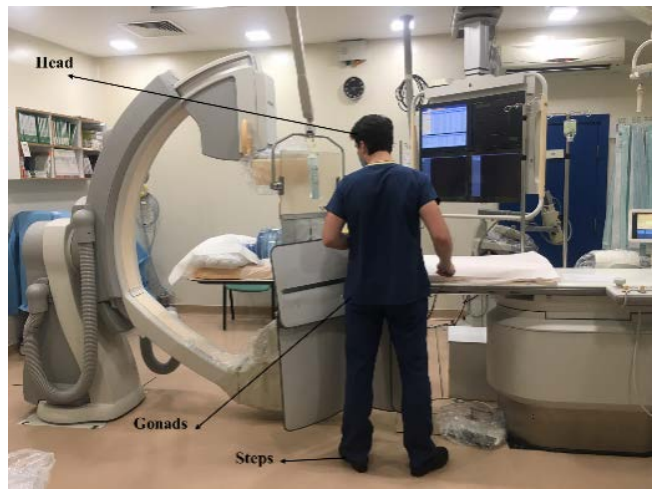

Figure 4. Position of cardiologist in relation to patient table, G-arm, radiation protection shield. Measuring points: head, gonads and steps. 
Table 1. Projections for LAD-stenting.

\begin{tabular}{cc}
\hline AP & $0^{\circ} / 0^{\circ}$ \\
\hline RAO-cranial & $30^{\circ} / 30^{\circ}$ \\
LAO-cranial & $30^{\circ} / 30^{\circ}$ \\
Cranial & $30^{\circ}$ \\
\hline
\end{tabular}

Total time for the other projections-15 to $30 \mathrm{~min}$. The exposure time for these projections in pulse fluoroscopy is about 5 minutes.

2) LCx-stenting-Following projections are used:

Total time for the performed procedure 45 - $90 \mathrm{~min}$ (Table 2).

Basic projection-30 to $60 \mathrm{~min}$. Each of the three mentioned projections can be used as basic projection depending on the procedure. The exposure time in pulse fluoroscopy mode for the basic projection is about $10 \mathrm{~min}$. The exposure time in radiographic mode for the basic projection is about $2-3 \mathrm{~min}$.

Total time for the other projections-15 to $30 \mathrm{~min}$. Exposure time for these projections in fluoroscopy mode is about 5 minutes.

3) RCA-stenting-Following projections are used:

Total time for the performed procedure about $120 \mathrm{~min}$ (Table 3).

First basic projection-LAO $30^{\circ}$-about $60 \mathrm{~min}$. Exposure time in pulse fluoroscopy mode about $10 \mathrm{~min}$. The exposure time in radiography mode is about 2 -3 minutes.

Second basic projection RAO-cranial $30^{\circ} / 30^{\circ}-$ about $30 \mathrm{~min}$. The exposure time in pulse fluoroscopy mode is about 5 minutes. The exposure time in radiography mode is about $1 \mathrm{~min}$.

Second basic projection-Cranial $30^{\circ}$-about $30 \mathrm{~min}$. The exposure time in pulse fluoroscopy mode is about 5 minutes. The exposure time in radiography mode is about $1 \mathrm{~min}$.

The calculations are done for each of the projections as basic projection and for the remaining two projections as non-basic projections [5].

\section{Results}

Figure 5 shows comparative diagrams of the received dose load for the whole procedure for each of the three performed procedures, for the three patient table positions and at the three measurement points. The diagram is composed of the diagrams LAD-stenting, LCx-stenting and RCA-stenting. For each of the procedures, the dose received in fluoroscopic and radiographic mode of operation is calculated. The calculations are performed first for 2 minutes radiographic mode and then for 3 minutes radiographic mode for the basic projection.

For this reason "2 minutes" and " 3 minutes" values are shown in the diagrams. For the procedures LAD-stenting and LCx-stenting the fluoroscopy doses are summed for the respective duration for the three used projections and the 2 minutes radiographic mode of the basic projection; for the RCA-stenting the 
doses are summed of the fluoroscopy mode and 1 minute radiographic mode for the second and the third projection and 2 minute radiography for the first basic projection. In the " 3 minutes" values, for the procedures LAD-stenting and LCx-stenting the doses are summed for the respective duration for the three used projections and the 3 minutes radiographic mode of the basic projection; for the RCA-stenting the doses are summed of the fluoroscopy mode and 1 minute radiographic mode for the second and the third projection and 2 minute radiography for the first basic projection.

Comparing the diagrams of Figure 5 it can be seen that the RCA-stenting procedure shows the highest values of the received dose. This can easily be explained as this procedure runs the longest under X-ray control. The received dose is almost the same for all positions in the patient table. The highest value for the measurement point "Gonads", in all positions of the patient table in the range between 70 and $100 \mu \mathrm{Sv}$ can be clearly seen, more pronounced for the 3-minute radiographic mode of the first basic projection.

The lowest value is obtained for the measurement point "Head", namely about $50 \mu \mathrm{Sv}$ in 3 minutes radiography mode and about $40 \mu \mathrm{Sv}$ in 2 minutes radiography mode for the first basic projection RAO-cranial. The largest differences in the dose values are observed for the measurement point "Feet" - in the range 50 $\mu \mathrm{Sv}$ to $80 \mu \mathrm{Sv}$.

Because the other two projections, namely LAD-stenting and LCx-stenting, show almost same exposure time, the dose values will be compared for them (Figure 5).

When comparing the doses obtained at different positions of the patient table, lowest dose values are observed for the lowest position of the table. This is observed for these two procedures and for 2 minutes radiography and 3 minutes radiography in the main projection. In the other two positions of the patient table, there is no clear advantage concerning the dose load. At the highest and zero table position, higher values are indicated by the LAD-stenting. This procedure shows a higher value, in general, compared to the $\mathrm{LCx}$-stenting procedure.

Table 2. Projections for LCx-stenting.

\begin{tabular}{cc}
\hline AP & $0^{\circ} / 0^{\circ}$ \\
\hline RAO-caudal & $30^{\circ} / 15^{\circ}$ \\
LAO-caudal & $30^{\circ} / 30^{\circ}$ \\
Cranial & $30^{\circ}$ \\
\hline
\end{tabular}

Table 3. Projections for RCA-stenting.

\begin{tabular}{cc}
\hline AP & $0^{\circ} / 0^{\circ}$ \\
\hline RAO-cranial & $30^{\circ} / 30^{\circ}$ \\
LAO-cranial & $30^{\circ} / 30^{\circ}$ \\
Cranial & $30^{\circ}$ \\
\hline
\end{tabular}



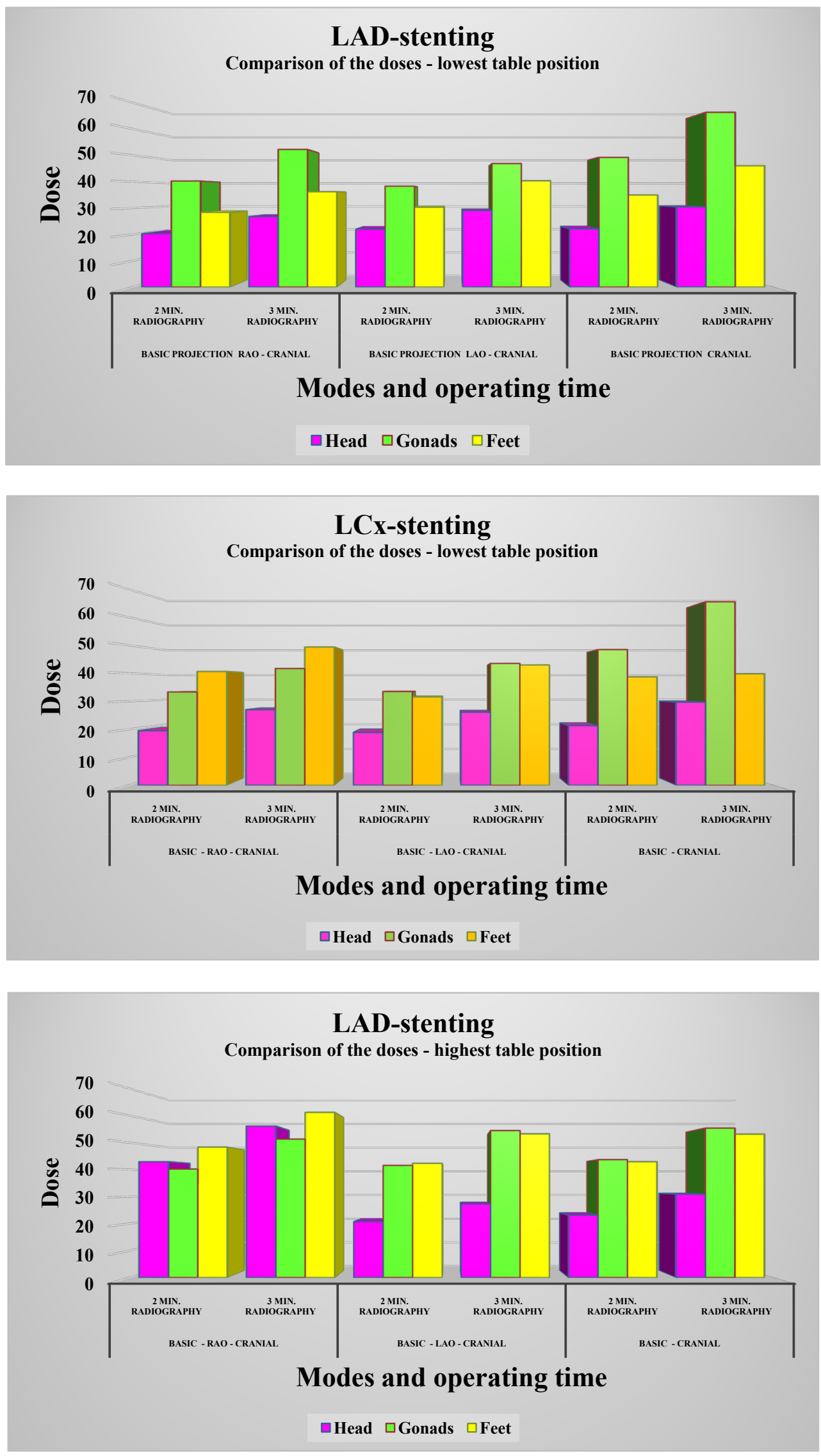

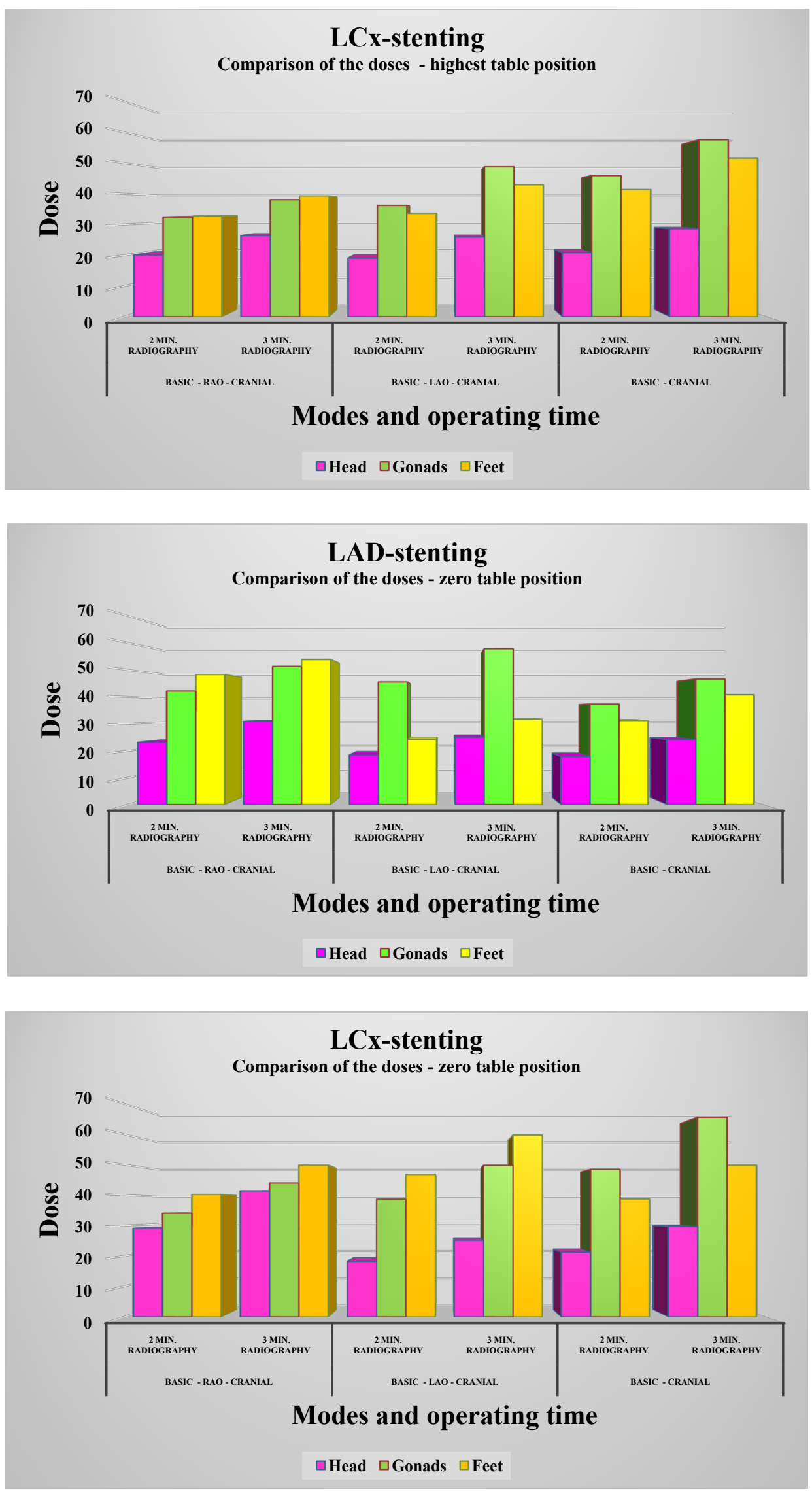


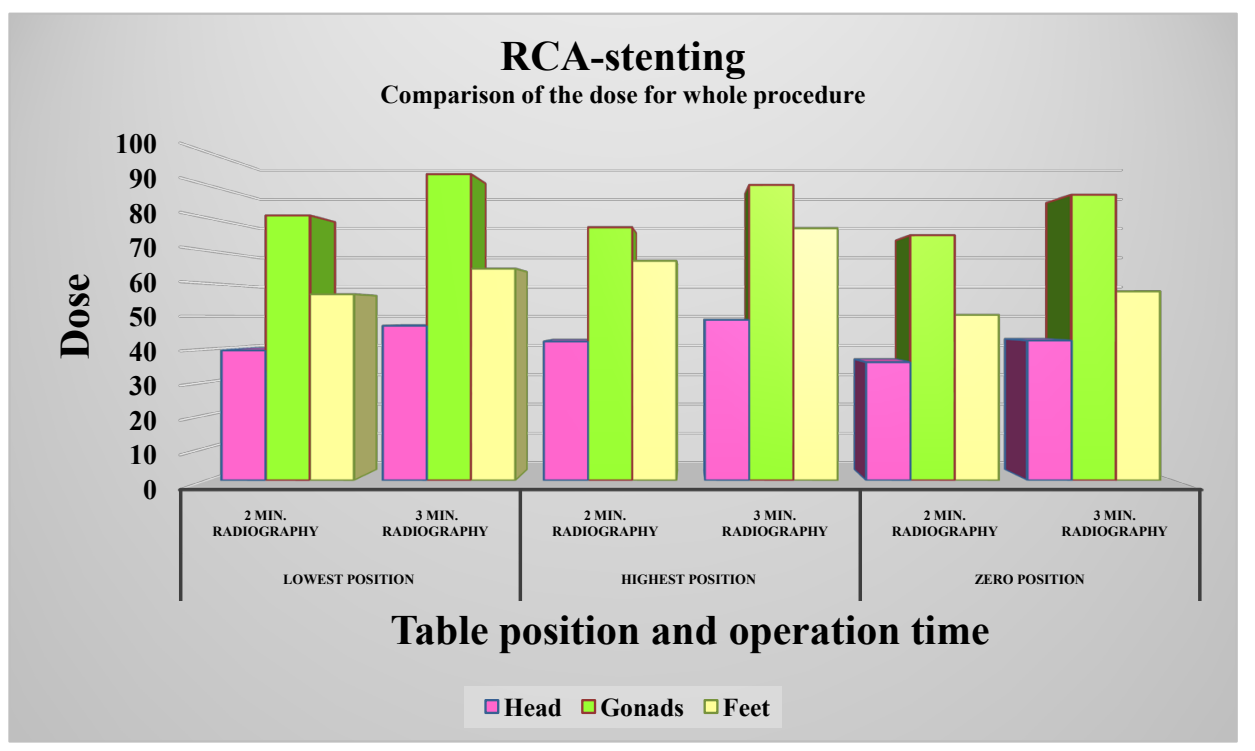

Figure 5. Comparison of the doses received by the first operator, at three points of his body, for the duration of the entire procedure for the three procedures, for different basic projections and in three different positions of the patient table.

Comparing the dose load in the different measurement points, the minimum dose is observed clearly outlined for the "Head" point for all procedures. Values equal to and higher than $40 \mu \mathrm{Sv}$ are observed only in the highest position of the patient table for the RAO-cranial projection, when this projection is chosen as basic projection in the LAD-stenting procedure. A lower dose load between 30 $\mu \mathrm{Sv}$ and $40 \mu \mathrm{Sv}$ for the measurement point "Head" is observed in the zero position of the patient table for RAO-caudal projection, when this projection is chosen as basic for the procedure LCx-stenting. The lowest dose values for the measurement point "Head"-below $20 \mu \mathrm{Sv}$-are observed for 2 minutes radiography and below $30 \mu \mathrm{Sv}$ for 3 minutes radiography of the basic projection.

The dose load at the measurement point "Steps" is in the range from $30 \mu \mathrm{Sv}$ to $50 \mu \mathrm{Sv}$ for 2 minutes radiography or $40 \mu \mathrm{Sv}$ to $60 \mu \mathrm{Sv}$ for 3 minutes radiography of the basic projection [5].

Most pronounced values were obtained for this measurement point for both projections, namely $60 \mu \mathrm{Sv}$ for LAD-stenting in the basic projection RAO-cranial and for the RCx-stenting with basic projection RAO-caudal. Predominantly, the values for the measurement point "Feet" for all procedures in different projections were measured between $30 \mu \mathrm{Sv}$ and $40 \mu \mathrm{Sv}$. The lowest value about $20 \mu \mathrm{Sv}$ was obtained for LAD-stenting in zero position of the table and 2 minutes radiography for the basic projection LAO-cranial.

The highest dose values for the duration of the whole procedure were obtained for the measurement point "Gonads". The maximum values for all procedures and all patient table positions are between $60 \mu \mathrm{Sv}$ and $70 \mu \mathrm{Sv}$ for $3 \mathrm{mi}-$ nutes radiography in the basic projection. Three of them are near $60 \mu \mathrm{Sv}$ when the patient table is in the highest and zero position of the procedure LAD-stenting and the highest position of the patient table for the procedure 
RCx-stenting. The other three are near $70 \mu \mathrm{Sv}$-for the lowest position of the table for the procedure $\mathrm{LAD}$-stenting and lowest and zero position for $\mathrm{RCX}$-stenting. The lowest values are between $35 \mu \mathrm{Sv}$ and $40 \mu \mathrm{Sv}$. Here most of these values are obtained for 2 minutes radiography for the basic projection for all procedures. Some values about $35 \mu \mathrm{Sv}$ were observed for all positions of the table and the LAD-stenting. About $40 \mu \mathrm{Sv}$ were observed for RCx-stenting.

Comparing the measured values for the different positions of the patient table, for the different projections of each procedure maximum dose values are observed for the main projection. This is due to the fact that in the basic projection the longest exposure duration is used. In addition, both angiographic modes are used here-fluoroscopy and radiography while only fluoroscopy is used during the non-basic operating modes.

The diagrams show greater dose load from the radiography exposure of the basic projection compared to the fluoroscopy of the same projection, although the fluorography time is $10 \mathrm{~min}$ and the radiography time 2 or 3 minutes. The dose load due to the non-basic projections is minimal because for them also the exposure time is shorter 2.5 minutes for each basic projection and the used mode is pulse fluorography which is less heavy [5].

\section{Conclusions}

From the obtained results a definite conclusion about the dose load can be given only for RCA-stenting. In this procedure, the dose load is greatest at the different measurement points and for all positions of the patient table.

The dose load in the other two procedures is almost the same with small differences for the different positions of the patient table. It cannot be concluded definitely which one is more dose-loading. Differences in dose load are observed for the different patient table positions.

The results obtained from the calculation of the dose load for the three studied procedures show the following:

$>$ The highest dose load is obtained for the procedure RCA-stenting because the exposure time for this procedure is the longest:

- Fluoroscopy time 10 minutes for the first basic projection;

- Radiography time 2 or 3 minutes for the first basic projection;

- Fluoroscopy time 5 minutes per each projection for the second and third basic projection;

- Radiography time 1 minute per each projection for the second and third basic projection.

The total exposure time for this procedure is 24 minutes including 2 minutes radiography for the first basic projection or 25 minutes including 3 minutes radiography for the basic projection.

> Next lower dose load is obtained for the procedure LAD-stenting. Here, the dose load is higher than the dose load for the LCx-stenting even when the total exposure time is the same. The exposure time for both procedures is as follows: 
- Fluoroscopy time 10 minutes for the first basic projection;

- Radiography time 2 or 3 minutes for the first basic projection;

- Fluoroscopy for the non-basic projections-total exposure time 5 minutes for both projections.

The total exposure time for this procedure is 17 minutes including 2 minutes radiography for the basic projection or 18 min when 3 minutes radiography for the basic projection is used.

$>$ The lowest dose load was obtained for the LCx-stenting, which had the same duration of the exposure as the LAD-stenting procedure.

Comparing the exposure times of the three examined procedures, the reported difference of 7 minutes of the procedure RCA-stenting leads to increase of the dose load for this procedure.

The total exposure time for RCA-stenting is $4 \mathrm{~min}$, including $2 \mathrm{~min}$ radiography exposure time for the first basic projection or $5 \mathrm{~min}$ if the exposure time for the first basic projection is $3 \mathrm{~min}$. For the remaining two projections the radiography exposure time is 2 or $3 \mathrm{~min}$. These additional 2 minutes radiography lead to additional increase of the dose load for the RCA stenting compared to the procedures LAD-stenting and LCx-stenting.

The higher dose for the RCA-stenting is also due to the larger fluoroscopy exposure time during the second and third basic projection. Compared to the two other projections the fluoroscopy exposure time for the heaviest procedure is about $1 / 4$ times longer (20 min for RCA-stenting and $15 \mathrm{~min}$ for the other two procedures). Although to a lesser extent due to radiography, the RCA-stenting shows a higher dose due to the longer duration of the fluoroscopy [5].

\section{Ethics Declaration}

During the above research no living objects (animals or humans) were used as the object of study. A phantom (Figure 3) was used as the object of irradiation and the dose measurements were made at the positions in which the head, gonads and feet of a cardiologist would be located, as shown in Figure 4. There were no real human subjects in these positions and the measuring device was placed on a suitable holder. The X-ray equipment was operated by the service engineer who is maintaining this equipment, protected with all necessary individual radiation protection accessories-radiation protection apron with a lead equivalent of $0.5 \mathrm{~mm}$, placed in two layers on the front of the body, radiation protection belt fixing the apron, radiation protection goggles and radiation protection hat. During the exposure, the engineer stayed as far away from the X-ray equipment as possible. The tests were performed after the official consent and permission of the director of Cardiac Hospital, Prof. Nikolay Penkov, MD. and three invasive cardiologists, members of the research team, namely Dr. Ismet Taksinov, Dr. Hrisimir Todorov and Dr. Nikolai Alexandrov (these three physicians initiated our research). We have their consent to publish the results of the research. The service engineer of the X-ray equipment used for the tests, Eng. Yavor Ivanov, is a co-author of this article and therefore his approval of this 
publication is implied.

An employee of Philips provided us with information material about the $\mathrm{X}$-ray angiographic equipment (per internet), thus allowing us to use them in our work.

Based on these studies, the first author of the article Natasha Ivanova, ch. assistant in the Department of Physics and Biophysics at Varna Medical University, wrote a monograph "Angiographic X-ray system with G-arm. Radiation load of the staff”, published by the Medical University Varna in November 2020. The material for this article is based on this monograph, cited in the respective reference as the last in the list.

\section{Acknowledgements}

- We express our great gratitude to the Specialized Hospital for Active Treatment in Cardiology, Varna and especially to its director and the staff, for the permission to conduct our study and for the invaluable help and support they provided us.

- We also express our gratitude to Philips, which supported us in our research, providing us with the necessary materials and photos used in the article.

\section{Conflicts of Interest}

The authors declare no conflicts of interest regarding the publication of this paper.

\section{References}

[1] Strauss, K.J. and Seibert, A.J. Angiographic Equipment Selection and Configuration with Authors. https://radiologykey.com/angiographic-equipment-selection-and-configuration/

[2] Sadick, V., Reed, W., Collins, L., Sadick, N., Heard, R. and Robinson, J. (2010) Impact of Biplane versus Single-Plane Imaging on Radiation Dose, Contrast Load and Procedural Time in Coronary Angioplasty. British Journal of Radiology, 83, 379-394. https://doi.org/10.1259/bjr/21696839

[3] Mavrikou, I., Kottou, S., Tsapaki, V. and Neofotistou, V. (2008) High Patient Doses in Interventional Cardiology Due to Physicians' Negligence: How Can They Be Prevented? Radiation Protection Dosimetry, 12, 67-70.

https://doi.org/10.1093/rpd/ncn005

[4] Fornell, D. (2016) 5 Technologies to Reduce Cath Lab Radiation Exposure. https://www.dicardiology.com/article/5-technologies-reduce-cath-lab-radiation-exp osure

[5] Ivanova, N. (2020) Angiographic X-Ray System with G-Arm. Dose Load of Personnel-Monograph, Medical University, Varna. [Иванова, Н. (2020) Ангиографска рентгенова система с G-рамо. Дозово натоварване на персонала, Медицински университет, Варна, ноември. (In Bulgarian)]

[6] Röntgen-Gamma Dosimeter 27091, Technical Description and Operating Instructions, 2 September 2008.

http://www.step-sensor.de/media/main/rgd_27091-manual_.pdf 